Contract No. and Disclaimer:

This manuscript has been authored by Savannah River Nuclear Solutions, LLC under Contract No. DE-AC09-08SR22470 with the U.S. Department of Energy. The United States Government retains and the publisher, by accepting this article for publication, acknowledges that the United States Government retains a non-exclusive, paid-up, irrevocable, worldwide license to publish or reproduce the published form of this work, or allow others to do so, for United States Government purposes. 
Ground Water Technical Commentary

\title{
Effective Porosity Implies Effective Bulk Density in Sorbing Solute Transport
}

\author{
G. P. Flach
}

Savannah River National Laboratory

The concept of an effective porosity is widely used in solute transport modeling to account for the presence of a fraction of the medium that effectively does not influence solute migration, apart from taking up space. This non-participating volume or ineffective porosity plays the same role as the gas phase in single-phase liquid unsaturated transport: it increases pore velocity, which is useful towards reproducing observed solute travel times. The prevalent use of the effective porosity concept is reflected by its prominent inclusion in popular texts, e.g., de Marsily (1986), Fetter (1988, 1993) and Zheng and Bennett (2002).

The purpose of this commentary is to point out that proper application of the concept for sorbing solutes requires more than simply reducing porosity while leaving other material properties unchanged. More specifically, effective porosity implies the corresponding need for an effective bulk density in a conventional single-porosity model. The reason is that the designated non-participating volume is composed of both solid and fluid phases, both of which must be neglected for consistency. Said another way, if solute does not enter the ineffective porosity then it also cannot contact the adjoining solid. Conceptually neglecting the fluid portion of the non-participating volume leads to a lower (effective) porosity. Likewise, discarding the solid portion of the non-participating volume inherently leads to a lower or effective bulk density. In the author's experience, practitioners virtually never adjust bulk density when adopting the effective porosity approach.

Effective bulk density is easily derived in terms of assumed effective porosity. The following exercise assumes that the participating and non-participating volumes have the same pore scale porosity and solid density, but that is not required. Let $V=$ total volume, $V_{f}=$ fluid volume, $\varphi=V_{f} / V=$ porosity, $M_{s}=$ solid mass, $\rho_{s}=M_{s} /\left(V-V_{f}\right)=$ solid density, $\rho_{b}=M_{s} / V=(1-\varphi) \rho_{s}=$ bulk density, $V_{p}=$ participating (mobile) volume, and $f_{p}=V_{p} / V=$ participating fraction. Then the effective (participating, mobile) porosity is defined by

$$
\theta_{e f f}=\frac{V_{f p}}{V}=\frac{V_{f p}}{V_{f}} \frac{V_{f}}{V}=f_{p} \varphi
$$

where $V_{f p}$ is the fluid volume within the participating volume. Similarly the effective bulk density is defined by

$$
\rho_{b, e f f}=\frac{M_{s p}}{V}=\frac{M_{s p}}{M_{s}} \frac{M_{s}}{V}=f_{p} \rho_{b}=f_{p}(1-\varphi) \rho_{s}
$$


where $M_{s p}$ is the solid mass within the participating volume. Combining Equations 1 and 2 produces

$$
\rho_{b, e f f}=\frac{\theta_{e f f}}{\varphi} \rho_{b}=\frac{\theta_{e f f}}{\varphi}(1-\varphi) \rho_{s}
$$

One can also define an effective solid density, which is useful for modeling software that takes (or requires) solid density as input. Using Equations 2 and 3 the result is

$$
\rho_{s, e f f}=\frac{\rho_{b, e f f}}{\left(1-\theta_{e f f}\right)}=\frac{\theta_{e f f}}{\left(1-\theta_{e f f}\right)} \frac{(1-\varphi)}{\varphi} \rho_{S}
$$

We next examine the impact of alternative density assignments on solute retardation. To generate example values, we consider the following specific settings representative of a sedimentary aquifer at the Savannah River Site: $\varphi=0.40, \theta_{\text {eff }}=0.25$ (Flach et al. 2004), and $\rho_{b}=$ $1.6 \mathrm{~g} / \mathrm{cm}^{3}$. The sorption coefficient $\left(K_{d}\right)$ is arbitrarily assumed to be $1.0 \mathrm{~cm}^{3} / \mathrm{g}$.

As one intuitively anticipates, Equation 3 preserves retardation between the total $(R)$ and effective porosity $\left(R_{\text {eff }}\right)$ systems

$$
R=1+\frac{\rho_{b} K_{d}}{\varphi}=1+\frac{\rho_{b, e f f} K_{d}}{\theta_{e f f}}=R_{e f f}=5
$$

In contrast, if the unaltered original bulk density is used with an effective porosity in forward model predictions, then retardation is biased high

$$
1+\frac{\rho_{b} K_{d}}{\theta_{\text {eff }}}=7.4>1+\frac{\rho_{b} K_{d}}{\varphi}=5
$$

The bias is larger still if the unaltered solid density is coupled with an effective porosity

$$
1+\frac{\left(1-\theta_{\text {eff }}\right) \rho_{s} K_{d}}{\theta_{\text {eff }}}=9>1+\frac{(1-\varphi) \rho_{s} K_{d}}{\theta_{\text {eff }}}=1+\frac{\rho_{b} K_{d}}{\theta_{\text {eff }}}=7.4>1+\frac{\rho_{b} K_{d}}{\varphi}=5
$$

If experimental retardation data are fit using a single porosity model with variable effective porosity but bulk density fixed at the total porosity value, then the apparent sorption coefficient will be biased low because the analysis assumes excess solid is present

$$
\frac{\theta_{e f f}(R-1)}{\rho_{b}}=0.625<\frac{\theta_{e f f}(R-1)}{\rho_{b, e f f}}=K_{d}=1
$$

Thus the direction of the bias differs for inverse modeling versus forward simulations. Biases are zero when effective porosity is equal to total porosity, and increase with increasing nonzero ineffective porosity. These modeling biases can be eliminated by adopting an effective bulk density using Equation 3. 


\section{References}

60 de Marsily, G. 1986. Quantitative Hydrogeology: Groundwater Hydrology for Engineers.

61 Orlando: Academic Press, 440 p.

62 Fetter, C. W. 1988. Applied Hydrogeology. Second edition. New York: Macmillan Publishing

63 Company, $592 \mathrm{p}$.

64 Fetter, C. W. 1993. Contaminant Hydrogeology. New York: Macmillan Publishing Company, 458

65 p.

66 Flach, G.P., S.A. Crisman, and F.J. Molz III. 2004. Comparison of single-domain and dual-domain

67 subsurface transport models. Ground Water 42, no. 6: 815-828.

68 Zheng, C., and G.D. Bennett. 2002. Applied Contaminant Transport Modeling. Second edition.

69 New York: John Wiley and Sons, $621 \mathrm{p}$. 